\title{
Title: Unexpected rewards induce dopamine-dependent positive emotion-like state changes in bumblebees
}

3 Authors: Clint J. Perry ${ }^{*}$, Luigi Baciadonna, Lars Chittka

5 Affiliations:

$6 \quad{ }^{1}$ Department of Biological and Experimental Psychology, School of Biological and Chemical

7 Sciences, Queen Mary University of London, London E1 4NS, UK

* To whom correspondence should addressed: clint.perry@qmul.ac.uk

11 Abstract:

12 Whether invertebrates exhibit positive emotion-like states and the mechanisms underlying such

13 states remain poorly understood. We demonstrate that bumblebees exhibit dopamine-dependent

14 positive emotion-like states across behavioral contexts. After training on one rewarding and one

15 unrewarding cue, bees that received pre-test sucrose responded in a positive manner towards

16 ambiguous cues. In a second experiment, pre-test consumption of sucrose solution resulted in a

17 shorter time to re-initiate foraging after a simulated predator attack. These behavioral changes

18 were abolished with topical application of the dopamine-antagonist fluphenazine. Further

19 experiments establish that pre-test sucrose is not simply causing bees to become more

20 exploratory. Our findings reveal a new opportunity to understanding the fundamental neural

21 elements of emotions and may alter our view of how emotion states affect decision-making in

22 animals.

23 Main Text:

24 Emotions are transient subjective states, underpinned by physiological, behavioral and

25 cognitive phenomena, triggered by appraisal of environmental situations (1-3). Our conceptual

26 understanding of emotion is largely based on human subjective experiences, i.e. what we 'feel',

27 assessed directly through verbal reports. In animals, similar emotion-like states can be inferred 
28 through observable, quantifiable parameters. To ensure the criteria of emotion-like states are

29 met, and to distinguish these from other forms of environmentally induced states, perhaps driven

30 by learning, we must quantify the range of physiological, behavioral and cognitive phenomena

31 that occur in response to environmental factors similar to those studied in humans (4).

The majority of work on animal emotions focuses on mammals and almost exclusively

33 on negative emotions (5). The idea that invertebrates may exhibit basic forms of emotion is

34 increasingly accepted (6-8), and given the assumed adaptive function of emotions (to coordinate

35 the individual's cognitive and behavioral resources towards fitness-relevant priorities $(1,2,9))$,

36 we might expect that a diversity of emotion-like states, including positive ones, exist across

37 phyla, albeit not necessarily consciously so (9-11).

In humans, consumption of sweet snacks can induce positive emotions (12-14). Here, we examine whether a small amount of pre-test sucrose solution causes bumblebees (Bombus

40 terrestris) to behave in a way indicative of an induced positive emotion-like state.

41 In Experiment 1.1, we utilized the well-established judgment bias paradigm, where

42 subjects associate one cue with a positive event and another cue with a negative event (15).

43 Subjects in a positive emotion state tend to respond to ambiguous (intermediate) stimuli as if 44 predicting the positive event (4).

45 We trained bees on a go/no-go task, to enter a cylinder beneath a colored placard (e.g.

46 blue) on one side of an arena where they would find $30 \%$ sucrose solution (Fig. 1A-B). On

47 alternate trials, bees learned to not enter a cylinder at the opposite side of the arena under a

48 placard of different color without reward (e.g. green, water). The latency from the time bees

49 entered the arena to the time they entered the presented cylinder was recorded. 
We then examined bees' response ('judgment') to ambiguous information (intermediate

51 color and location; Fig. 1C). Half of the trained bees, randomly selected, found for the first time,

52 and drank, a $5 \mu$ l droplet (equivalent to $<5 \%$ of stomach capacity) of $60 \%$ sucrose solution in the

53 tunnel leading to the arena or received no reward. Bees that consumed sucrose solution prior to

54 making a decision took less time to enter the chamber of the middle $(\mathrm{M})$ ambiguous stimulus

55 (Fig. 1D; Tables S1-S2; Supplementary Materials).

Could it be that when a bee consumed the small reward, rather than a positive emotion-

57 like state, a higher expectation of subsequent reward resulted in greater exploration of novel

58 stimuli? Indeed, previous work indicates that honeybees' foraging choices are controlled by short

59 term memories initiated by rewards just experienced $(16,17)$. However, bees tested on stimuli

60 not intermediate to the trained stimuli (novel in terms of color, position and number; Experiment

61 1.2; Fig. 1E), exhibited no difference in choice time (Fig. 1F; Table S3) or number of choices

62 (Fig. 1G; Table S4) between conditions, indicating that pre-decision sucrose consumption did not

63 cause a general increase in expectation of reward.

64 Consumption of sucrose solution may simply make bees more excited or active, resulting

65 in faster decisions for ambiguous stimuli. Thorax temperature increased after consumption of $5 \mu 1$

$6660 \%$ sucrose solution $\left(\mathrm{n}=72, \mathrm{t}_{70}=6.78, \mathrm{p}=3.12 \mathrm{e}^{-9}\right.$; Experiment 2.1; Fig. S1 A,B; Supplemental

67 Materials) denoting increased metabolic rate. But this did not transfer to increased activity.

68 Sucrose and control bees $(n=24)$ showed no difference in flight time $\left(\mathrm{t}_{22}=0.666, \mathrm{p}=0.512\right)$ or

69 speed $\left(\mathrm{t}_{22}=0.241, \mathrm{p}=0.812\right)$ to a feeder (Experiment 2.2; Fig. S1 C, D; Supplemental

70 Materials), and when the feeder was removed, speed during a 120s flight also did not differ

71 between groups $\left(n=24, t_{22}=-0.403, p=0.691\right.$; Experiment 2.3; Fig. S1 E), suggesting that

72 unexpected rewards did not affect bees' overall activity level. 
It has been argued that one characteristic of emotions across species is generalization - a

74 property whereby an induced emotion state operates across behavioral contexts (9). To examine

75 whether these behavioral results were similar across contexts we tested whether an unanticipated

76 reward would change bees' reaction to later aversive stimuli (Experiment 3). We trained bees to

77 forage at a feeder containing $30 \%$ sucrose solution. After training and on their next foraging trip,

78 bees were held temporarily in the tunnel connecting the hive and arena. Bees would either

79 receive an unanticipated $5 \mu 1$ droplet of $60 \%$ sucrose solution or nothing (control). After a 10-

80 second delay, a predator attack was simulated. At natural flowers, bees are sometimes ambushed

81 by sit-and-wait predators such as crab spiders; bees often escape after a brief struggle, allowing

82 them to modify their subsequent behavior to cope with such threat (18). Mimicking such an

83 attack, the bee was captured by a trapping mechanism, applying constant pressure for three

84 seconds by a stamp-shaped device softened with a sponge connected to a micro-servo (Fig. 2A;

85 (18)). The bee was subsequently released and the time it took to commence foraging was

86 recorded.

87 Sweet food can increase positive emotions and improve negative mood in human adults,

88 and reduce crying and grimacing of newborns in response to aversive stimuli (12-14). If

89 drinking an unexpected sucrose solution caused a positive emotion-like state in bees, we predict

90 that, following consumption, bees' aversive reaction to the 'predator' would be attenuated.

91 Indeed, bees that consumed sucrose solution prior to the 'attack' took less time to re-initiate

92 foraging $\left(n=35, t_{33}=-3.70, p=7.87 e^{-04}\right.$; Fig. $\left.2 B\right)$.

93 The insect reward system parallels that of mammals in several aspects, including some of

94 the neurochemicals involved (19). In mammals, several neurotransmitters play key roles in both

95 reward processing and emotions. We asked if the biogenic amines linked to reward processing in 
96 the insect brain might be involved in the behaviors suggestive of emotion-like states here

97 observed. We topically treated bees $(20,21)$ with antagonists of biogenic amines Octopamine

98 (OA; antagonist: mianserin; $\mathrm{n}=20$ ), Dopamine (DA; antagonist: fluphenazine; $\mathrm{n}=20$ ) and

99 Serotonin $(5-\mathrm{HT}$; antagonist: yohimbine; $\mathrm{n}=20)$ and determined their effect on behavior induced

100 by pre-decision sucrose. Bees were trained as in Experiment 3. Fifteen minutes after antagonist

101 application, or vehicle control (DMF; $\mathrm{n}=20)$, bees consumed, for the first time, a $5 \mu$ l droplet of

$10260 \%$ sucrose solution. Following this, bees were subjected to a simulated predator attack and the

103 time taken to return to foraging was recorded (Experiment 4.1). Only bees treated with the DA-

104 antagonist took longer to begin foraging than control bees (ANOVA: $\mathrm{n}=96, \mathrm{df}=4, \mathrm{~F}=3.48, \mathrm{p}$

$105=0.011$; Tukey posthoc: $\mathrm{p}=0.039$; Fig. $3 \mathrm{~A}$ ). We speculate that this is a consequence of brain

106 dopamine signals responding to unexpected reward (22-25). To ensure that the DA-antagonist

107 was not simply interacting with pathways mediating normal response to the aversive stimulus,

108 bees were topically treated with DA-antagonist without receiving pre-test sucrose. The time to

109 begin foraging for these bees was similar to both bees treated with DA-antagonist treated + pre-

110 decision sucrose and control bees receiving no pre-test sucrose $(n=16 ;$ Fig. $3 \mathrm{~A}$ and Fig. 2B:

111 Control).

112 We explored whether blocking DA had similar effects on the observed cognitive

113 consequences of pre-decision reward in the judgment bias paradigm. Bees were trained as in

114 Experiment 1.1 and then treated with either DA-antagonist or DMF 15 minutes prior to

115 consuming an unexpected $5 \mu \mathrm{l}$ of $60 \%$ sucrose solution and testing. Compared to control, DA-

116 antagonist-treated bees took longer to enter the middle (M) ambiguous stimulus chamber

117 (Experiment 4.2; Fig. 3B; Table S5-S6). 
119 (23), including motivation for reward (25) nutritional value of reward (22) and arousal (26). Our

120 results corroborate DA's role in the neuronal processes mediating reward signals, in bees. An

121 intriguing prospect of research would be whether similar circuits controlling wanting, hunger,

122 nutritional valuation and/or arousal underpin the emotion-like states indicated by our results in

123 bees.

The behaviors displayed by bumblebees in response to a small amount of pre-decision

125 sucrose conform to criteria commonly applied to mammals for internal emotion-like states

126 interacting with decision-making: positive judgment bias to ambiguous stimuli and attenuated

127 response to negative stimuli. Whether common neural processing features evolved independently

128 or an ancient role of biogenic amines evolved to serve similar functions, new findings (including

129 ours) support the hypothesis that the fundamental elements of emotion exist in many species (9).

130 Our results lend support to the notion that invertebrates may have states that fit the

131 criteria defining emotion $(1,9)$. The adaptive function of emotion is thought to be the integration

132 of information about environment and body to modulate decisions and behavior (9).

133 Understanding and investigating the basic features of emotion states will bring us a step closer to 134 determining the brain mechanisms underlying emotion across taxa.

\section{References and Notes:}

137 1. D. Nettle, M. Bateson, The Evolutionary Origins of Mood and Its Disorders. Curr. Biol. 22, 138 R712-R721 (2012).

139 2. J. LeDoux, Rethinking the emotional brain. Neuron. 73, 653-676 (2012).

140 3. M. Mendl, O. H. P. Burman, E. S. Paul, An integrative and functional framework for the study of animal emotion and mood. Proc. R. Soc. Lond. B Biol. Sci. 277, 2895-2904

142 (2010). 
143 4. E. S. Paul, E. J. Harding, M. Mendl, Measuring emotional processes in animals: the utility 144 of a cognitive approach. Neurosci. Biobehav. Rev. 29, 469-491 (2005).

145 5. B. L. Fredrickson, What Good Are Positive Emotions? Rev. Gen. Psychol. J. Div. 1 Am. 146 Psychol. Assoc. 2, 300-319 (1998).

147 6. W. T. Gibson et al., Behavioral responses to a repetitive visual threat stimulus express a 148 persistent state of defensive arousal in Drosophila. Curr. Biol. CB. 25, 1401-1415 (2015).

149 7. P. Fossat et al., Comparative behavior. Anxiety-like behavior in crayfish is controlled by $150 \quad$ serotonin. Science. 344, 1293-1297 (2014).

151 8. M. Bateson, S. Desire, S. E. Gartside, G. A. Wright, Agitated Honeybees Exhibit 152 Pessimistic Cognitive Biases. Curr. Biol. 21, 1070-1073 (2011).

153 9. D. J. Anderson, R. Adolphs, A Framework for Studying Emotions Across Phylogeny. Cell. $154 \quad$ 157, 187-200 (2014).

10. J. Panksepp, Affective Neuroscience: The Foundations of Human and Animal Emotions (Oxford University Press, 1998).

157 11. P. Winkielman, K. C. Berridge, Unconscious Emotion. Curr. Dir. Psychol. Sci. 13, 120158 (2004).

12. P. M. A. Desmet, H. N. J. Schifferstein, Sources of positive and negative emotions in food experience. Appetite. 50, 290-301 (2008).

13. M. Fernandez et al., Sucrose attenuates a negative electroencephalographic response to an aversive stimulus for newborns. J. Dev. Behav. Pediatr. JDBP. 24, 261-266 (2003).

14. M. Macht, J. Mueller, Immediate effects of chocolate on experimentally induced mood states. Appetite. 49, 667-674 (2007).

15. E. J. Harding, E. S. Paul, M. Mendl, Animal behaviour: Cognitive bias and affective state. Nature. 427, 312-312 (2004).

16. U. Greggers, R. Menzel, Memory dynamics and foraging strategies of honeybees. Behav.

168 Ecol. Sociobiol. 32, 17-29 (1993).

169 17. L. C. Nigel E Raine, Flower constancy and memory dynamics in bumblebees

170 (Hymenoptera: Apidae: Bombus). Entomol. Gen. 29, 179-199 (2007).

171 18. T. C. Ings, L. Chittka, Speed-accuracy tradeoffs and false alarms in bee responses to cryptic 172 predators. Curr. Biol. CB. 18, 1520-1524 (2008).

173 19. C. J. Perry, A. B. Barron, Neural Mechanisms of Reward in Insects. Annu. Rev. Entomol. $174 \quad$ 58, 543-562(2013). 
20. A. B. Barron et al., Comparing injection, feeding and topical application methods for treatment of honeybees with octopamine. J. Insect Physiol. 53, 187-194 (2007).

21. N. DiRienzo, D. R. McDermott, J. N. Pruitt, Testing the Effects of Biogenic Amines and Alternative Topical Solvent Types on the Behavioral Repertoire of Two Web-Building Spiders. Ethology. 121, 801-812 (2015).

22. W. Huetteroth et al., Sweet Taste and Nutrient Value Subdivide Rewarding Dopaminergic Neurons in Drosophila. Curr. Biol. 25, 751-758 (2015).

23. A. B. Barron, E. Søvik, J. L. Cornish, The Roles of Dopamine and Related Compounds in Reward-Seeking Behavior Across Animal Phyla. Front. Behav. Neurosci. 4 (2010).

24. E. Søvik, C. J. Perry, A. B. Barron, in Advances in Insect Physiology, A. Z. and C. F. Kent, Ed. (Academic Press, 2015), vol. 48 of Genomics, Physiology and Behaviour of Social Insects, pp. 189-226.

25. M. J. Krashes et al., A Neural Circuit Mechanism Integrating Motivational State with Memory Expression in Drosophila. Cell. 139, 416-427 (2009).

26. R. Andretic, B. van Swinderen, R. J. Greenspan, Dopaminergic Modulation of Arousal in Drosophila. Curr. Biol. 15, 1165-1175 (2005).

27. L. Morawetz, J. Spaethe, Visual attention in a complex search task differs between honeybees and bumblebees. J. Exp. Biol. 215, 2515-2523 (2012).

Acknowledgments: We would like to thank Eirik Søvik, Magda Osman, Jochen Zeil, Christian Nawroth, Alan McElligott and Andrew Barron for comments on earlier drafts, and two anonymous reviewers for their helpful critical feedback. We also thank Eirik Søvik for help with statistical analyses. C.J.P. was funded by a Marie Curie Postdoctoral Fellowship. L.B. was funded by a Queen Mary University of London Departmental Studentship. L.C. was supported by an ERC Advanced Grant and a Royal Society Wolfson Research Merit Award.

Author Contributions: L.B. conceived the study. C.J.P., L.B. and L.C. designed the experiments. C.J.P and L.B. conducted the experiments and carried out behavioral data analysis. The manuscript was written by C.J.P., L.B. and L.C. 


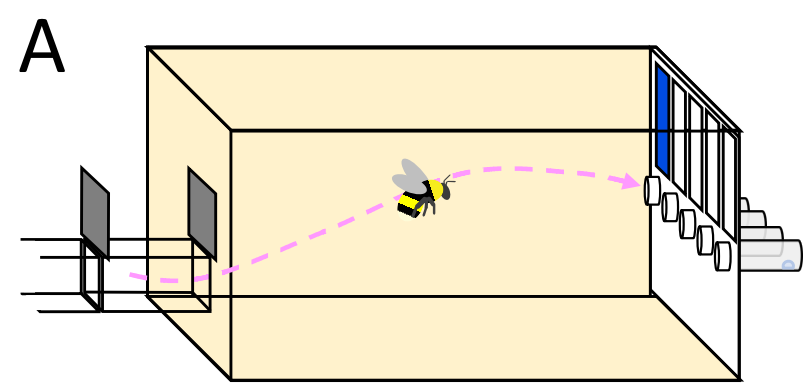

B

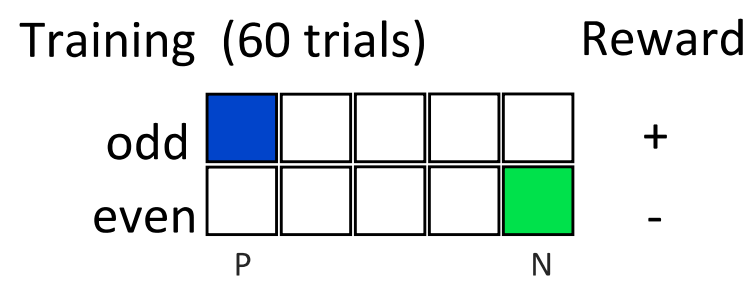

C

D
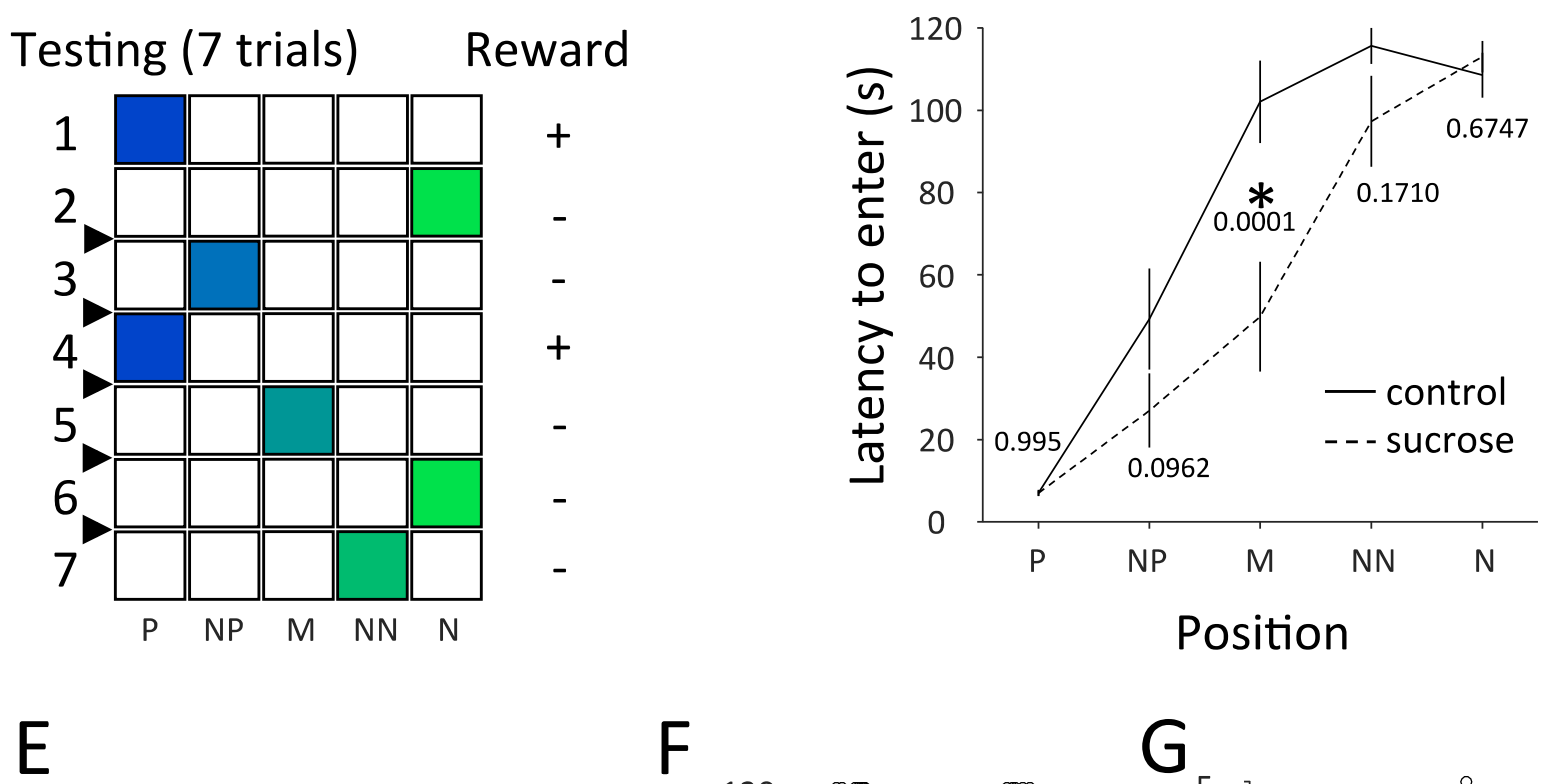

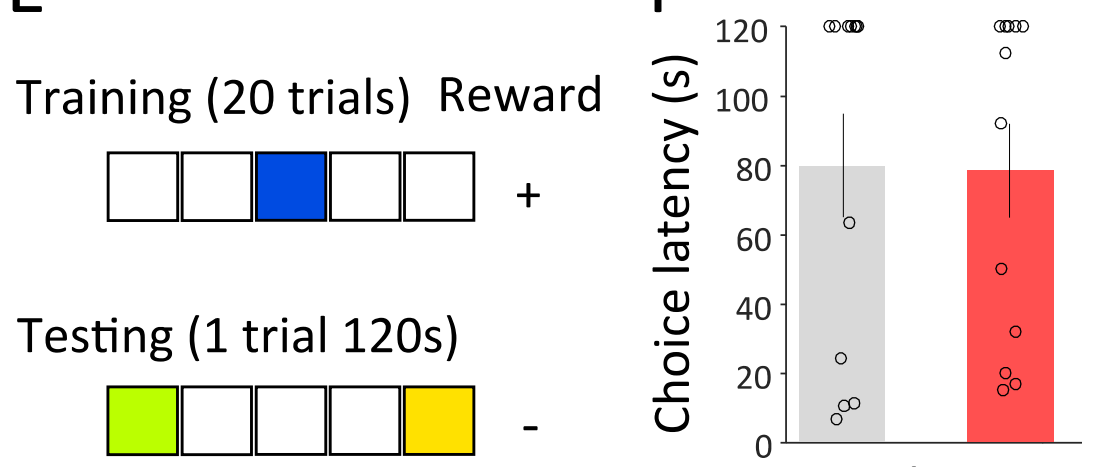

Control Sucrose

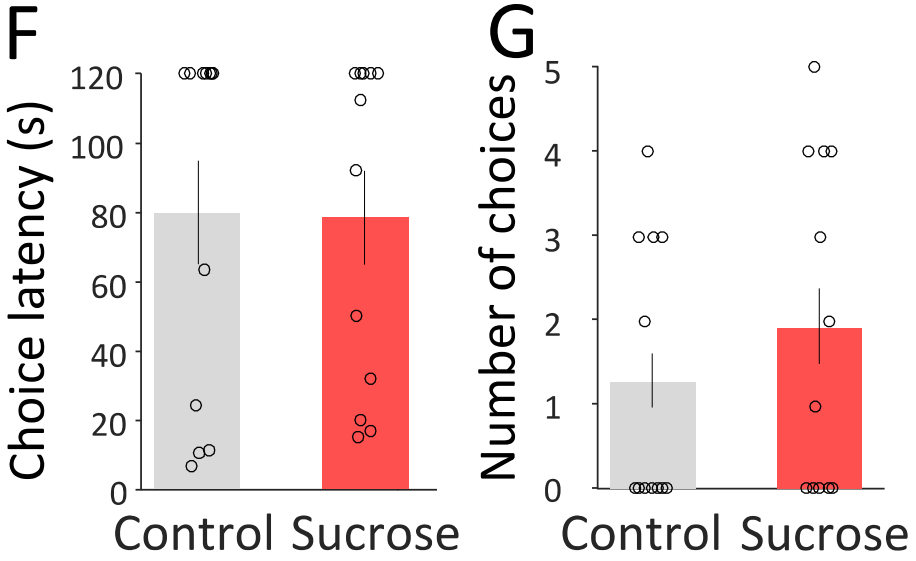

Fig. 1. Judgment bias to ambiguous stimuli. (A) Set up for Experiment 1.1. (B-C) Each row

209 shows a 'bee's eye view' of placards within arena. (B) Training stimuli for one of four

210 counterbalanced orientations (Fig. S2; $N=$ Negative, $\mathrm{P}=$ positive). Bees $(\mathrm{n}=24)$ were trained 
211 find sucrose solution in a cylinder under one placard and avoid another. Only one cylinder was

212 accessible on any one trial: odd trials were rewarded and even trials unrewarded. (C) Testing

213 procedure. Half the bees received pre-test sucrose (arrowheads). After two 'reminder trials', bees

214 were tested on three ambiguous stimuli alternated between trained stimuli. Order was

215 counterbalanced (Fig. S3). (D) Results of Experiment 1.1. The sucrose group took less time to

216 enter the middle position (M) than the control group. Numbers are p values. (E) Training

217 procedure for Experiment 1.2. Bees $(\mathrm{n}=24)$ were trained to find a reward under a blue placard,

218 and subsequently tested on two novel stimuli. (F-G) Results for Experiment 1.2. (F) Latency to

219 feeder and (G) number of choices did not differ between groups. Here and elsewhere, bars =

220 mean, open circles $=$ individual bees. Here and elsewhere, error bars $=$ s.e. Generalized linear

221 modeling analyses in Tables S1-S4. 

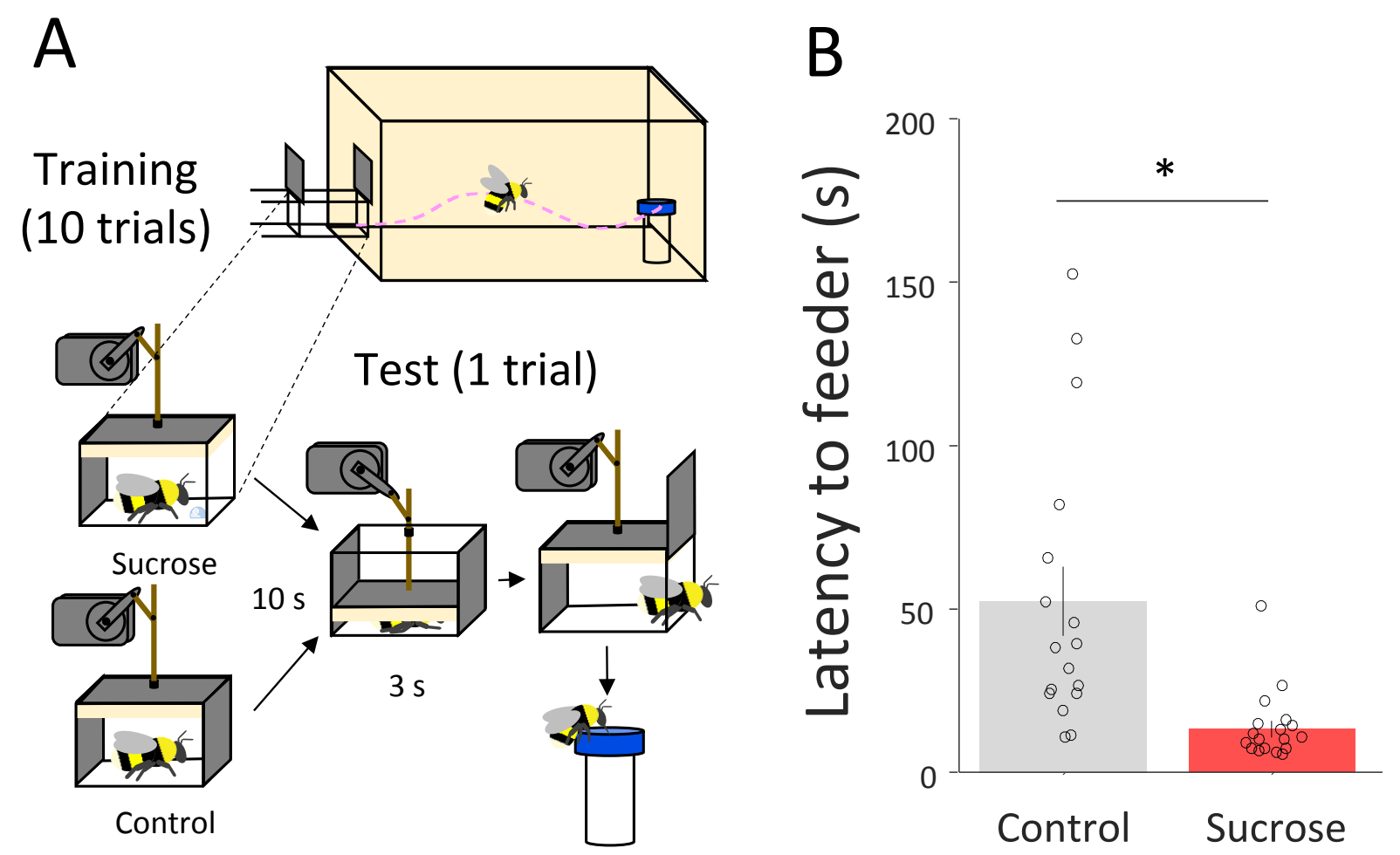

226 Fig. 2. Attenuation of response to aversive stimuli. (A) Training and test procedure for

227 Experiment 3. Bees $(n=35)$ were trained to feed at a $30 \%$ sucrose solution feeder. Subsequently,

228 bees consumed $5 \mu 1$ of $60 \%$ sucrose solution prior to a simulated predator attack. (B) Results of

229 predation experiment. Sucrose group bees took less time to resume foraging behavior than the

230 control group $\left(\mathrm{t}_{33}=-3.70, \mathrm{p}=7.87 \mathrm{e}^{-04}\right)$. 


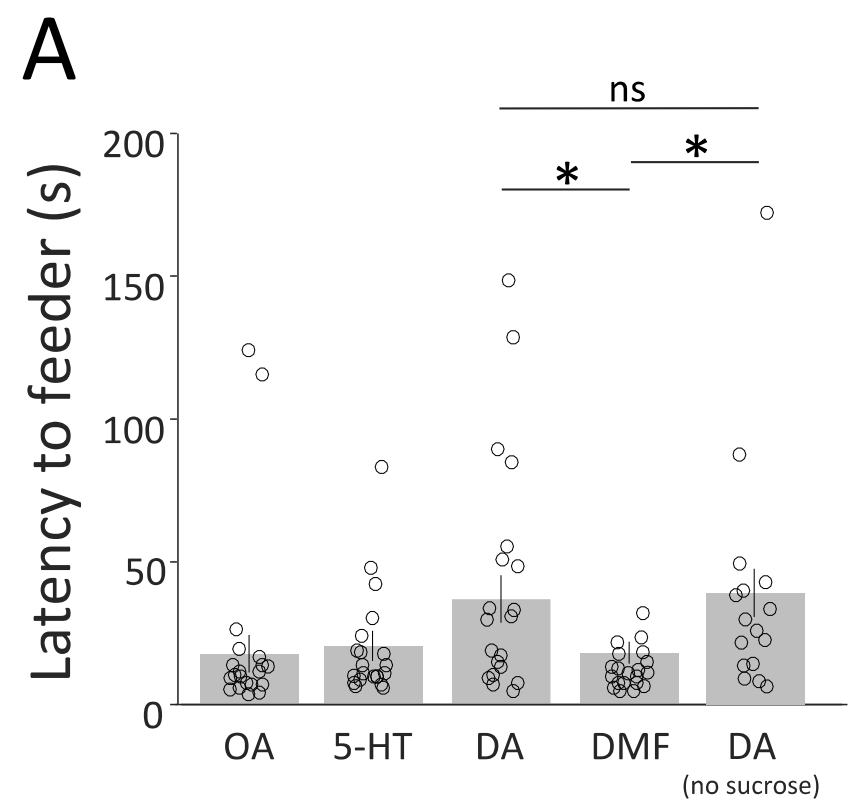

Treatments

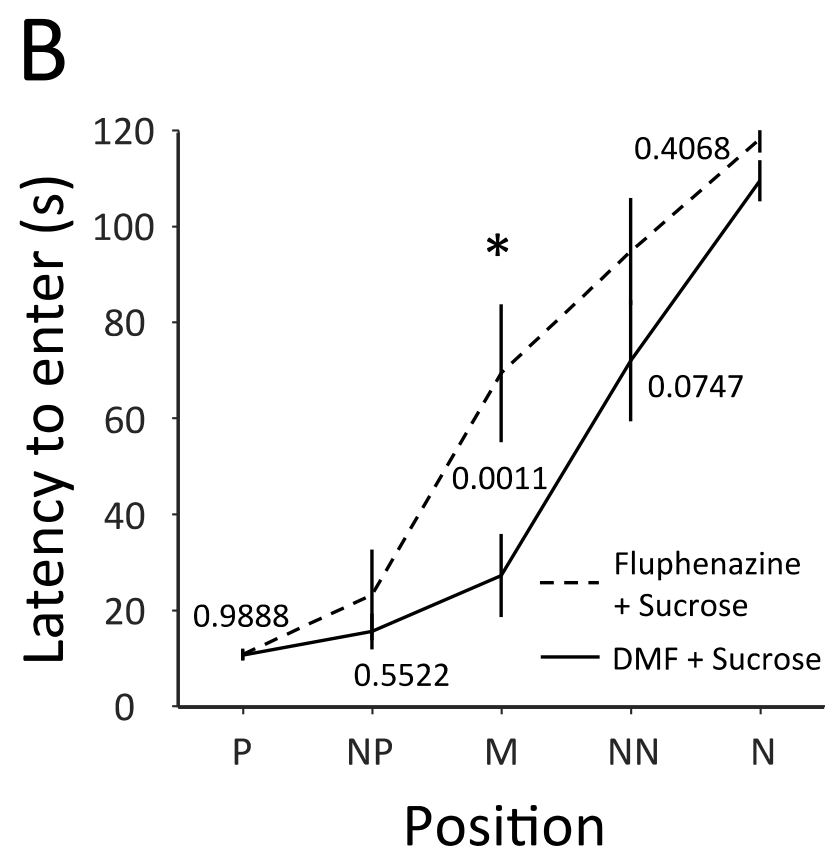

233 Fig. 3. Results of experiments blocking biogenic amines. (A) Results for Experiment 4.1:

234 Predation. Dopamine (DA), but not Octopamine (OA) or Serotonin (5-HT) antagonist-treated 235 bees took more time to resume foraging behavior than DMF-treated bees ( $\mathrm{n}=96$; $\mathrm{t}$-test with 
236 Bonferroni correction; DA: $\mathrm{t}_{38}=3.14, \mathrm{p}=0.003$; OA: $\mathrm{t}_{38}=1.19, \mathrm{p}=0.241,5-\mathrm{HT}: \mathrm{t}_{38}=1.16, \mathrm{p}=$

237 0.113). (B) Results for Experiment 4.2: Judgment Bias. Fluphenazine (DA-antagonist) treated

238 bees took more time to enter the middle position (M) than vehicle (DMF) treated bees. Numbers

239 indicate $\mathrm{p}$ values. Generalized linear modeling analyses in Tables S5-S6.

240

241 Supplementary Materials:

242 Materials and Methods

243 Figures S1-5

244 Tables S1-6

$245 \quad$ Reference 27 